\title{
DESVIACIONES AL MODELO LOGARÍTMICO-LINEAL EN LA SOLUBILIDAD DE IBUPROFÉN Y NAPROXÉN EN MEZCLAS COSOLVENTES PROPILENOGLICOL-AGUA
}

\section{Edgar F. Vargas}

Departamento de Química, Universidad de los Andes, Bogotá D. C., Colombia

Yady J. Manrique, Diana P. Pacheco, Norma S. Torres y Fleming Martínez*

Departamento de Farmacia, Universidad Nacional de Colombia, A.A. 14490, Bogotá D. C., Colombia

Recebido em 29/1/07; aceito em 11/5/07; publicado na web em 16/10/07

\begin{abstract}
DEVIATIONS FROM LOG-LINEAR SOLUBILITY EQUATION FOR IBUPROFEN AND NAPROXEN IN PROPYLENE GLYCOL-WATER COSOLVENT MIXTURES. The deviations observed in the solubility of ibuprofen (IBP) and naproxen (NAP) in propylene glycol $(\mathrm{PG})+$ water $(\mathrm{W})$ cosolvent mixtures with respect to the logarithmic-linear model proposed by Yalkowsky have been analyzed at $25.00 \pm 0.05^{\circ} \mathrm{C}$. Negative deviations were obtained in all cosolvent compositions for both drugs; they were greater for IBP. Another treatment, based on Gibbs free energy relationships, was also employed showing an apparent hydrophobicity chameleonic effect, because at low PG proportions NAP is more hydrophobic, whereas at high PG proportions IBP is more hydrophobic. The results are discussed in terms of solute-solvent and solvent-solvent interactions.
\end{abstract}

Keywords: AINEs; cosolvent mixtures; log-linear solubility equation.

\section{INTRODUCCIÓN}

El ibuprofén (IBP) y el naproxén (NAP) son fármacos del tipo analgésico-anti-inflamatorios, derivados del ácido propiónico, y clasificados como anti-inflamatorios no esteroidales (AINEs), que son ampliamente utilizados en la terapéutica actual para el tratamiento sintomático de la artritis reumatoidea y en general, para todo proceso agudo o crónico relacionado con dolor e inflamación ${ }^{1}$. En el mercado farmacéutico colombiano, el IBP se dispone comercialmente en forma de tabletas y suspensiones para administración peroral, mientras que el NAP se presenta como tabletas, cápsulas, polvo para suspensión, y además como suspensión preparada, para administración peroral. Así mismo, el IBP no se dispone para administración parenteral, mientras que el NAP si se presenta como inyectable en ampollas de $500 \mathrm{mg} / 5 \mathrm{~mL}^{2}$.

De otro lado, es bien sabido que las formulaciones líquidas inyectables se caracterizan por suministrar una alta dosis de fármaco en un pequeño volumen de producto; por lo tanto, algunas propiedades fisicoquímicas tales como la solubilidad y los volúmenes ocupados por los principios activos y los otros componentes en la solución se tornan muy importantes para el diseñador farmacéutico, ya que el conocimiento de estas propiedades, así como el adecuado manejo y de ser posible, la predicción de estos fenómenos, facilita enormemente la labor de este profesional durante su labor en el desarrollo de medicamentos ${ }^{3}$.

Por lo anteriormente expuesto, específicamente en lo relacionado con la predicción de propiedades de los sistemas líquidos, en la presente investigación se presenta un estudio fisicoquímico sobre el efecto de la composición cosolvente sobre la solubilidad del IBP y el NAP a $25,00 \pm 0,05{ }^{\circ} \mathrm{C}$ en mezclas binarias solventes formadas por propilenoglicol (PG) y agua (W). En particular, sobre las desviaciones presentadas por estos dos sistemas respecto al modelo logarítmico-lineal de Yalkowsky y Roseman ${ }^{4}$.

El sistema PG-W es de amplio uso en el diseño de formulaciones líquidas de administración parenteral ${ }^{4,5}$ y además, ha sido muy bien

*e-mail: fmartinezr@unal.edu.co estudiado desde el punto de vista físicoquímico, en particular, los volúmenes molares parciales y de exceso ${ }^{6}$ y las entalpías molares de exceso ${ }^{7}$. Así mismo, los aspectos termodinámicos de solución ${ }^{8}$ y la predicción de la solubilidad de algunos otros fármacos en este sistema binario han sido estudiados previamente ${ }^{9}$.

\section{El modelo logarítmico-lineal de solubilidad}

En la literatura se han descrito diferentes métodos para estimar la solubilidad de varias clases de solutos en mezclas cosolventes, algunos de los cuales han sido ampliamente desafiados recientemente frente a la solubilidad experimental de ciertos fármacos ${ }^{10}$.

Uno de los modelos más simples para estimar esta propiedad en mezclas cosolvente-agua está basado en la regla de la mezcla algebraica, la cual, para el caso de fármacos no polares en una mezcla binaria adquiere la forma:

$\log X_{2-\text { Mix }}=f \log X_{2-\text { Cosolv }}+(1-f) \log X_{2-\text { Water }}$

donde $X_{2-M i x}$ es la solubilidad calculada en la mezcla cosolvente considerada, $X_{2 \text {-Cosolv }}$ es la solubilidad en el cosolvente puro, $X_{2 \text {-Water }}$ es la solubilidad en agua pura, y $f$ es la fracción volumétrica del cosolvente, calculada asumiendo aditividad de volúmenes como $f$ $=V_{\text {Cosolv }} /\left(V_{\text {Cosolv }}+V_{\text {Water }}\right)$, en la que $V_{\text {Cosolv }}$ y $V_{\text {Water }}$ son los volúmenes de cosolvente y agua, respectivamente ${ }^{11}$. La Ecuación 1 es una forma práctica del modelo logarítmico-lineal desarrollado por Yalkowsky y que tiene la forma:

$\log S_{2-\text { Mix }}=\log S_{2-\text { Water }}+\sigma \cdot f$

donde $S_{2-\text { Mix }} y S_{\text {2-Water }}$ son las solubilidades (expresadas en molaridad o fracción molar) en la mezcla cosolvente y en el agua, respectivamente, y $\sigma$ es el factor de potencia cosolvente-solubilizante del respectivo sistema soluto-solvente. El término $\sigma$ ha sido correlacionado con diferentes índices de polaridad de los solventes, tales como el coeficiente de reparto octanol-agua, el parámetro de solubilidad de Hildebrand, y las tensiones interfaciales, entre otros ${ }^{12}$. 
La ecuación 2 ha sido ampliamente utilizada en el estudio de diversos solutos de interés farmacéutico ${ }^{4,13}$.

Sin embargo, experimentalmente se ha encontrado que el comportamiento de diferentes solutos lipofílicos se desvía de esta sencilla regla aditiva, en particular si los cosolventes son anfipróticos. Específicamente, en el caso del sistema PG-W, Rubino y Obeng ${ }^{11}$ estudiando la solubilidad de series homólogas de phidroxibenzoatos y p-aminobenzoatos de alquilo, encontraron desviaciones negativas a la Ecuación 1 en mezclas ricas en agua, esto es, a bajas fracciones volumétricas de PG, y desviaciones positivas en las mezclas ricas de cosolvente. Puesto que estos investigadores encontraron cierta similaridad en las desviaciones encontradas por los diferentes solutos, han sugerido que las interacciones cosolvente-agua son las principales responsables de estos comportamientos ${ }^{11}$.

De acuerdo con esto, se plantea que los cosolventes pueden interactuar con el agua principalmente mediante dos mecanismos: (a) la naturaleza orgánica de los cosolventes lleva a un tipo particular de interacción con el agua, la cual ha sido descrita como la formación de "témpanos" de agua alrededor de las porciones apolares del cosolvente, esto es, la hidratación hidrofóbica. Puesto que este fenómeno depende de la capacidad del agua de formar uniones cohesivas con otras moléculas de agua, es de esperar que este tipo de interacción sea predominante a bajas concentraciones de cosolvente, en las cuales se tiene presente un exceso de moléculas de agua. Este fenómeno ha sido descrito por Kimura et al. ${ }^{14}$ en el estudio de las entalpías de transferencia de la 1-metil-2pirrolidinona desde el agua hasta algunos alcoholes de cadena corta. (b) En adición a los efectos hidrofóbicos, los grupos hidroxilo del PG pueden interactuar con el agua mediante enlaces de hidrógeno, por lo que se ha sugerido que a bajas concentraciones de los alcoholes de cadena corta, estos pueden aumentar la estructuración del agua a través de enlaces de hidrógeno, en adición al efecto hidrofóbico. Por lo tanto, los dos tipos de interacciones mencionadas anteriormente, que llevan a un aumento en la estructuración de agua, conducen a su vez a una disminución en las interacciones soluto-solvente, según se ha demostrado mediante estudios de calor de disolución en estos sistemas ${ }^{14}$. De otro lado, cuando la fracción de PG es alta, los enlaces de hidrógeno entre el cosolvente y el agua siguen estando presentes pero la estructuración del agua ha disminuido significativamente o ha desaparecido.

\section{PARTE EXPERIMENTAL}

\section{Reactivos}

Ibuprofén USP ${ }^{15}$; naproxén USP ${ }^{15}$; propilenoglicol USP ${ }^{15}$ deshidratado (PG); agua destilada, conductividad $<2 \mu \mathrm{S} \mathrm{cm}$, alcohol USP ${ }^{15}$; tamiz molecular Merck (números 3 y 4); filtros Durapore ${ }^{\circledR}$ 0,45 $\mu \mathrm{m}$ Millipore Corp.; material aforado de vidrio.

\section{Preparación de las mezclas cosolventes}

Se prepararon mezclas binarias PG-W de composición desde 0,00 hasta $100,00 \% \mathrm{~m} / \mathrm{m}$ de $P G$ variando de 10,00 en $10,00 \% \mathrm{~m} / \mathrm{m}$, por pesada en una balanza digital de platillo externo, Mettler Toledo PB302 de sensibilidad $\pm 0,01 \mathrm{~g}$, estudiando así la solubilidad en los dos solventes puros y en nueve mezclas solventes.

\section{Determinación de la densidad}

La densidad de las mezclas cosolventes y de las soluciones saturadas se determinó utilizando un densímetro digital DMA 45 Anton
Paar conectado a un termostato Magni Whirl Blue M a 25,00 \pm 0,05 ${ }^{\circ} \mathrm{C}$ según procedimiento descrito previamente. El densímetro se calibró según las instrucciones del catálogo del equipo. Las soluciones se introdujeron en el tubo de vibración del equipo utilizando una jeringa plástica de $2 \mathrm{~mL}$ esperando hasta la estabilización de la lectura de densidad en la pantalla digital (precisión $\pm 0,0001 \mathrm{~g} \mathrm{~cm}^{-3}$ ) ${ }^{16}$.

\section{Determinación de la solubilidad}

Se colocaron cantidades específicas de las diferentes mezclas PG-W y de los solventes puros en frascos de vidrio NP (No parenteral: Tipo $\mathrm{IV}^{15}$ ) y en cada uno se adicionó un exceso de IBP o NAP, se taparon los frascos, se agitaron mecánicamente en un agitador Burell ${ }^{\circledR}$ Modelo 75 durante $1 \mathrm{~h}$ y se colocaron en el baño termostatado a $40,00 \pm 0,05{ }^{\circ} \mathrm{C}$ con agitación manual esporádica durante al menos $72 \mathrm{~h}$ para acelerar el proceso de saturación. Posteriormente se ajustó la temperatura en $25,00 \pm 0,05{ }^{\circ} \mathrm{C}$ dejando las muestras durante al menos $24 \mathrm{~h}$ para permitir la precipitación del exceso de fármaco disuelto. Se tomaron muestras de las soluciones sobrenadantes, las cuales fueron filtradas para eliminar partículas sólidas no disueltas, determinándoles la densidad siguiendo el procedimiento mencionado anteriormente, para realizar la interconversión entre unidades másicas y volumétricas. Las muestras fueron diluidas gravimétricamente con alcohol USP ${ }^{15}$ y analizadas por espectrofotometría UV en un equipo BioMate 3 Thermo Electron Corp. siguiendo metodologías analíticas validadas ${ }^{17}$. Todos los procedimientos indicados se realizaron al menos tres veces y los respectivos resultados fueron promediados.

\section{RESULTADOS Y DISCUSIÓN}

En la Tabla 1 se presentan los valores de algunas propiedades fisicoquímicas de los fármacos en estudio, tomados de la literatura, mientras que en la Tabla 2 se presenta la solubilidad de los dos fármacos, expresada en molaridad y en fracción molar (en la mayoría de los casos el coeficiente de variación en solubilidad fue menor del 3,0\%), observándose claramente que la solubilidad experimental se incrementa de forma no lineal con el incremento en la proporción de PG en las mezclas cosolventes.

De la misma forma se observa que en todos los casos la solubilidad experimental del IBP es mayor que la del NAP, lo cual es consistente con lo esperado de acuerdo a la solubilidad ideal ( 0,268 y 0,0833 para IBP y NAP a $25,0{ }^{\circ} \mathrm{C}$, respectivamente $\left.{ }^{20,21}\right)$; esto es, de acuerdo con las propiedades físicas de los fármacos en el estado sólido, ya que las entalpías y las temperaturas de fusión son mayores para el NAP respecto al IBP (Tabla 1) y por lo tanto, la tendencia hacia el estado líquido es mayor en el caso del IBP. Es necesario aclarar que bajo las condiciones cosolventes estudiadas aquí, la disociación de estos dos fármacos es mínima, con lo que se tiene que la solubilidad total presentada por éstos, corresponde prácticamente en su totalidad a la de la forma molecular no disociada, y por lo tanto se trataría de la solubilidad intrínseca ${ }^{13}$.

En la Figura 1 se presenta la solubilidad experimental del IBP y del NAP expresada como el cociente logarítmico respecto a la solubilidad en agua. Los valores se presentan de esta forma para poder evaluar el efecto relativo de la hidrofobicidad de los solutos sobre las tendencias respectivas. En los dos casos se obtienen polinomios regulares de tercer orden con coeficientes de determinación $\mathrm{r}^{2}$ de 0,9998 y 0,9999 para el IBP y el NAP, respectivamente. A diferencia de lo reportado por Rubino y Obeng ${ }^{11}$ con p-hidroxibenzoatos de alquilo, en los cuales, en todas las composiciones cosolventes, el cociente resultó mayor para los derivados de cadena más larga y por lo tanto más hidrofóbicos, en el 
Tabla 1. Algunas propiedades físicas y químicas de IBP y NAP

\begin{tabular}{|c|c|c|c|c|c|c|}
\hline Fármaco & Estructura molecular (a) & $\mathrm{M} / \mathrm{g} \mathrm{mol}^{-1}$ (a) & $\Delta H_{f} / \mathrm{kcal} \mathrm{mol}^{-1}$ & $T_{\text {fus }} / \mathrm{K}$ & $\mathrm{pK}_{\mathrm{a}}(\mathrm{b})$ & $\lambda_{\text {máx }} / \mathrm{nm}(\mathrm{c})$ \\
\hline IBP & 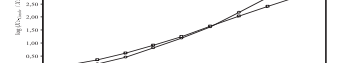 & 206,28 & $6,09(d)$ & $347,15(d)$ & 5,2 & 265 \\
\hline NAP & & 230,26 & 7,53(e) & $427,6(\mathrm{e})$ & 4,2 & 271 \\
\hline
\end{tabular}

(a) Tomado de Budavari et al..$^{18}$; (b) Tomado de Betageri et al. ${ }^{19}$; (c) Determinado en alcohol USP ${ }^{15}$; (d) Tomado de Ertel et al..$^{20}$; (e) Tomado de Perlovich et al. ${ }^{21}$.

Tabla 2. Solubilidad de IBP y NAP a $25,0^{\circ} \mathrm{C}$ expresada en molaridad y en fracción molar

\begin{tabular}{lccccc}
\hline & PG & \multicolumn{2}{c}{ IBP } & $X_{2}$ & NAP \\
$\% \mathrm{~m} / \mathrm{m}$ & $f$ & Mol L & $\mathrm{L}^{-1}$ & $X_{2}$ \\
\hline 0,00 & 0,0000 & $7,544(0,003) \times 10^{-4}$ & $1,360(0,006) \times 10^{-5}$ & $2,84(0,12) \times 10^{-4}$ & $5,10(0,21) \times 10^{-6}$ \\
10,00 & 0,0969 & $8,339(0,014) \times 10^{-4}$ & $1,619(0,028) \times 10^{-5}$ & $4,00(0,21) \times 10^{-4}$ & $7,70(0,40) \times 10^{-6}$ \\
20,00 & 0,1944 & $1,037(0,003) \times 10^{-3}$ & $2,180(0,006) \times 10^{-5}$ & $5,47(0,23) \times 10^{-4}$ & $1,16(0,05) \times 10^{-5}$ \\
30,00 & 0,2926 & $1,725(0,003) \times 10^{-3}$ & $3,947(0,006) \times 10^{-5}$ & $9,30(0,03) \times 10^{-4}$ & $2,13(0,08) \times 10^{-5}$ \\
40,00 & 0,3916 & $3,134(0,005) \times 10^{-3}$ & $9,056(0,011) \times 10^{-5}$ & $1,68(0,03) \times 10^{-3}$ & $4,24(0,10) \times 10^{-5}$ \\
50,00 & 0,4912 & $8,104(0,004) \times 10^{-3}$ & $2,095(0,011) \times 10^{-4}$ & $3,24(0,05) \times 10^{-3}$ & $9,14(0,15) \times 10^{-5}$ \\
60,00 & 0,5915 & $1,772(0,004) \times 10^{-2}$ & $5,690(0,012) \times 10^{-4}$ & $7,05(0,03) \times 10^{-3}$ & $2,26(0,05) \times 10^{-4}$ \\
70,00 & 0,6925 & $5,46(0,04) \times 10^{-2}$ & $2,051(0,015) \times 10^{-3}$ & $1,47(0,01) \times 10^{-2}$ & $5,49(0,04) \times 10^{-4}$ \\
80,00 & 0,7943 & $0,1698(0,0027)$ & $7,77(0,12) \times 10^{-3}$ & $2,92(0,02) \times 10^{-2}$ & $1,31(0,01) \times 10^{-3}$ \\
90,00 & 0,8968 & $0,4642(0,0011)$ & $2,770(0,007) \times 10^{-2}$ & $5,40(0,09) \times 10^{-2}$ & $3,02(0,05) \times 10^{-3}$ \\
100,00 & 1,0000 & $1,133(0,004)$ & $9,85(0,04) \times 10^{-2}$ & $9,72(0,05) \times 10^{-2}$ & $7,26(0,04) \times 10^{-3}$ \\
\hline
\end{tabular}

caso del IBP y del NAP se tiene que esta situación es variable dependiendo de la composición cosolvente; esto es, por debajo de la fracción 0,6 de PG el efecto solubilizante es mayor para el NAP, mientras que por encima de esta proporción de PG la situación se invierte, lo cual aparentemente estaría significando un cambio en las magnitudes de las hidrofobicidades aparentes relativas, esto es, por debajo de $f$ 0,6 el NAP sería más lipofílico que el IBP y por encima de este valor el comportamiento sería contrario.

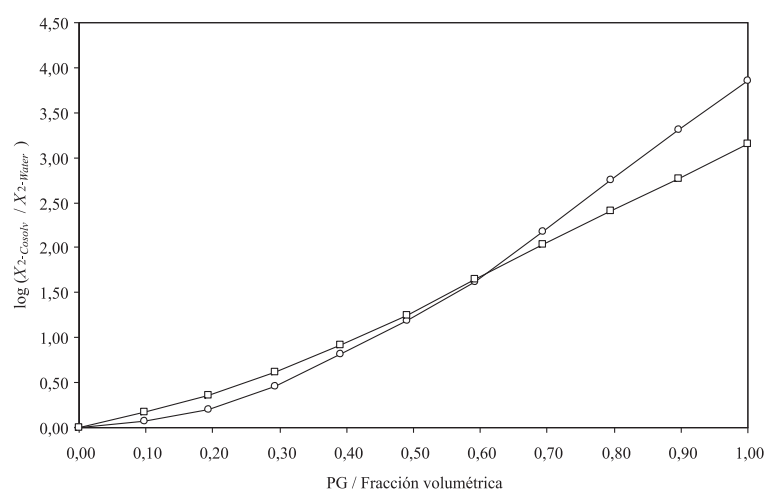

Figura 1. Variación del log $\left(\mathrm{X}_{2 \text {-Cosolv }} / \mathrm{X}_{2 \text {-Water }}\right)$ en función de $f_{P G}$ para IBP $(\mathrm{O})$ y NAP ( $\square$ ) a $25,0^{\circ} \mathrm{C}$

Como un criterio de lipofilicidad tradicional para estos compuestos, se compararon los valores calculados de coeficiente de reparto octanol-agua reportados por Burgot y Burgot ${ }^{22}$, expresados como $\log K_{o / w}$, esto es, 4,50 y 3,51, para el IBP y el NAP respectivamente. Así mismo se comparó el coeficiente de distribución entre el octanol y una solución acuosa regulada a $\mathrm{pH}$ 7,0 (presentado como $\log D_{o / w}$ por Betageri et al. ${ }^{19}$ ) para el IBP, esto es, 1,88 , el cual al ser corregido por efecto del $\mathrm{pH}^{23}$ (mediante la Ecuación 3) se transforma en 3,70 (expresado como log $K_{o / w}$ ), frente al valor 3,50 para el NAP (expresado también como $\log K_{o / w}$ ) presentado por $\mathrm{Mora}^{24}$.

$K_{\mathrm{o} / \mathrm{w}}=D_{\mathrm{o} / \mathrm{w}}\left(1+10^{\mathrm{pH}-\mathrm{pKa}}\right)$

De acuerdo a los valores anteriores se podría considerar que el IBP es más hidrofóbico que el NAP, o expresado en otros términos, que el IBP es menos polar que el NAP, lo cual se corrobora al calcular el parámetro de solubilidad de Hildebrand $\delta$ para los dos compuestos mediante el método de contribución de grupos de Fedors $^{25}$ como se muestra en la Tabla 3, obteniendo los valores 10,22 $\mathrm{cal}^{1 / 2} \mathrm{~cm}^{-3 / 2}$ y $10,94 \mathrm{cal}^{1 / 2} \mathrm{~cm}^{-3 / 2}$ para el IBP y el NAP, respectivamente. Los valores $\delta$ representan la raíz cuadrada de la densidad de energía cohesiva y son por lo tanto, un índice de polaridad, esto es, a mayor valor de $\delta$ más polar es el compuesto 9 .

Otra aproximación al establecimiento de hidrofobicidades relativas está dada en términos de las relaciones lineales de energía libre, que aplicadas al caso de solubilidad podrían interpretarse así: considerando el equilibrio heterogéneo que se establece cuando un exceso de sólido cristalino (Fármaco A) está en contacto con un solvente $\mathrm{S} 1$, a $T$ y $P$ constantes, la concentración del fármaco A alcanzada es su solubilidad $\left(X_{A}\right)$ en $\mathrm{S} 1$ y este valor es constante en esas condiciones.

$\mathrm{Al}$ alterar el equilibrio anterior agregando un solvente miscible con $\mathrm{S} 1$, se produce una nueva condición solvente $\mathrm{S} 2$, frente a la cual el sistema responde aumentando o disminuyendo la solubilidad 
Tabla 3. Aplicación del método de Fedors para la estimación de la energía interna, el volumen molar y el parámetro de solubilidad de Hildebrand de IBP y NAP

\begin{tabular}{|c|c|c|c|}
\hline Grupo & $\begin{array}{c}\text { IBP } \\
\text { Cantidad }\end{array}$ & $\mathrm{E} / \mathrm{cal} \mathrm{mol}^{-1}$ & $\mathrm{~V} / \mathrm{cm}^{3} \mathrm{~mol}^{-1}$ \\
\hline $\mathrm{COOH}$ & 1 & 6600 & 28,5 \\
\hline $\mathrm{CH}_{3}$ & 3 & $3 \times 1125=3375$ & $3 \times 33,5=100,5$ \\
\hline $\mathrm{CH}_{2}$ & 1 & 1180 & 16,1 \\
\hline $\mathrm{CH}^{2}$ & 2 & $2 \times 820=1640$ & $2 \times-1,0=-2,0$ \\
\hline Fenilo disustituido & 1 & 7630 & 52,4 \\
\hline Total & & 20425 & 195,5 \\
\hline \multicolumn{4}{|c|}{$\delta=(20425 / 195,5)^{1 / 2}=10,22 \mathrm{cal}^{1 / 2} \mathrm{~cm}^{-3 / 2}$} \\
\hline Grupo o átomo & $\begin{array}{c}\text { NAP } \\
\text { Cantidad }\end{array}$ & $\mathrm{E} / \mathrm{cal} \mathrm{mol}^{-1}$ & $\mathrm{~V} / \mathrm{cm}^{3} \mathrm{~mol}^{-1}$ \\
\hline $\mathrm{O}$ & 1 & 800 & 3,8 \\
\hline $\mathrm{COOH}$ & 1 & 6600 & 28,5 \\
\hline $\mathrm{CH}_{3}$ & 2 & $2 \times 1125=2250$ & $2 \times 33,5=67,0$ \\
\hline $\mathrm{CH}$ & 1 & 820 & $-1,0$ \\
\hline$-\mathrm{C}=$ & 4 & $4 \times 1030=4120$ & $4 \times 13,5=54,0$ \\
\hline Fenilo trisustituido & 1 & 7630 & 33,4 \\
\hline Total & & 22220 & 185,7 \\
\hline
\end{tabular}

del fármaco $A$, según la naturaleza de este soluto y del cambio en las características del solvente. Esta variación en la solubilidad involucra un cambio en la energía libre del sistema $\left(\Delta G_{A(1 \rightarrow 2)}^{\mathrm{o}}\right)$ de acuerdo a:

$\Delta G_{A(1 \rightarrow 2)}^{o}=-2,303 R T\left(\log X_{A(S 2)}-\log X_{A(S 1)}\right)$

Analizando ahora el equilibrio de solubilidad de un segundo compuesto (Fármaco B), relacionado estructuralmente con el primero, el cual es a su vez perturbado de la misma forma como se hizo con el fármaco A, transfiriéndolo del solvente $\mathrm{S} 1$ al solvente $\mathrm{S} 2$, el nuevo equilibrio del fármaco $\mathrm{B}$ responderá de una forma similar al fármaco $\mathrm{A}$, aunque con una intensidad diferente ${ }^{26}$.

De forma específica, para dos fármacos cristalinos estructuralmente relacionados A y $\mathrm{B}$ en $n$ sistemas solventes se puede plantear la Tabla 4, con los consiguientes sistemas resultantes:

Tabla 4. Sistemas resultantes al disolver dos fármacos A y B en $n$ mezclas cosolventes

\begin{tabular}{lccccc}
\hline Fármacos & \multicolumn{5}{c}{ Solventes } \\
& $\mathrm{S} 1$ & $\mathrm{~S} 2$ & $\mathrm{~S} 3$ & $\ldots$ & $\mathrm{S} n$ \\
\hline $\mathrm{A}$ & $X_{A}(\mathrm{~S} 1)$ & $X_{A}(\mathrm{~S} 2)$ & $X_{A}(\mathrm{~S} 3)$ & $\ldots$ & $X_{A}(\mathrm{~S} n)$ \\
$\mathrm{B}$ & $X_{B}(\mathrm{~S} 1)$ & $X_{B}(\mathrm{~S} 2)$ & $X_{B}(\mathrm{~S} 3)$ & $\ldots$ & $X_{B}(\mathrm{~S} n)$ \\
\hline
\end{tabular}

Lo que se observa experimentalmente es que las correspondientes variaciones de energía libre $\Delta G_{A(1 \rightarrow 2)}^{\mathrm{o}}$ y $\Delta G_{B(1 \rightarrow 2)}^{\mathrm{o}}$ se relacionan linealmente. Las magnitudes experimentales correlacionables son los datos de $\log X_{A}$ en función de $\log X_{B}$, que corresponden a la solubilidad de los fármacos A y B en la misma serie de solventes $\mathrm{S} 1, \mathrm{~S} 2, \mathrm{~S} 3 \ldots$ Cuando se tienen relaciones lineales se llega a las siguientes expresiones:

$\log X_{B}=P_{b} \log X_{A}+c$

$\Delta \log X_{B}=P_{b} \Delta \log X_{A}$
En las cuales $P_{b}$ es el parámetro del efecto del medio solvente y caracteriza la respuesta relativa de las solubilidades a los cambios en el medio solvente. El término $P_{b}$ depende de la diferencia estructural entre los fármacos $\mathrm{A}$ y $\mathrm{B}$ y del efecto ocasionado por los cambios en el solvente sobre la solubilidad de ambos compuestos.

La Ecuación 5 ha sido aplicada satisfactoriamente a varios sistemas constituidos por series homólogas de sustratos de interés farmacéutico en agua, solventes hidroxílicos y mezclas de estos ${ }^{27}$.

Para interpretar estos resultados se desarrolló un modelo sencillo, denominado Modelo de la Condición de Máxima Solubilidad (MCMS), el cual permite el análisis y la predicción del efecto del medio sobre las solubilidades en equilibrio.

El MCMS considera a las moléculas de soluto y de solvente como la sumatoria de sus respectivos grupos hidrofílicos y lipofílicos, siendo este balance el que determina su solubilidad. Dentro de este contexto, una modificación estructural del fármaco A consistente en un aumento de su porción hidrocarbonada aumentaría su lipofilicidad, mientras que la introducción de un nuevo grupo hidrofílico, o el reemplazo de un grupo determinado por otro con un mayor carácter hidrofílico, aumentarían su hidrofilicidad.

Las conclusiones del efecto del medio en términos del MCMS pueden resumirse de la siguiente forma: dados dos fármacos A y B cuya solubilidad se determina en una serie de solventes de lipofilicidad creciente, entonces si un aumento en la lipofilicidad del solvente aumenta la solubilidad del fármaco A, el mismo cambio en el solvente producirá un aumento en la solubilidad del fármaco $\mathrm{B}$, que será mayor si éste es más lipofílico, y menor si es mas hidrofílico que el fármaco A. Por el contrario, cuando el aumento en la lipofilicidad del solvente produce una disminución en la solubilidad del fármaco A, el efecto sobre el fármaco B será menor si éste es más lipofílico y mayor si es más hidrofílico ${ }^{27}$.

En la Figura 2 se presenta la variación de $\log X$ del IBP respecto a la variación de $\log X$ del NAP obteniendo una regresión parabólica con valor $\mathrm{r}^{2}$ de 0,9993 , la cual al ser derivada y resuelta en cada punto de composición cosolvente permitió obtener los valores de pendiente $P_{b}$ presentados en la Tabla 5 . 


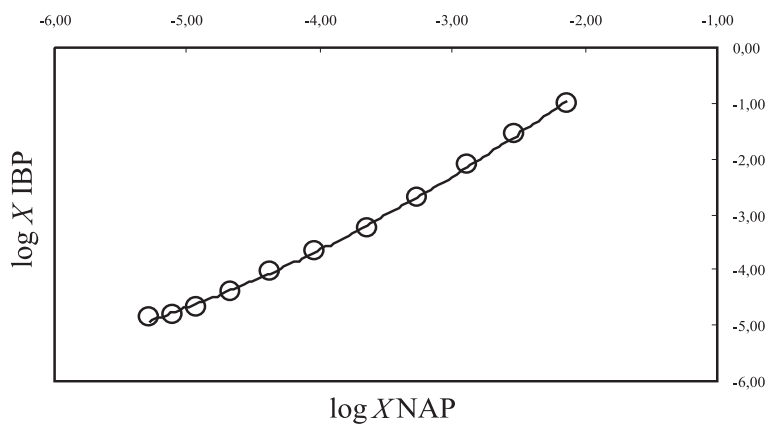

Figura 2. Variación del log $X$ de IBP en función de log X de NAP a $25,0{ }^{\circ} \mathrm{C}$

Tabla 5. Variación del parámetro del efecto del medio solvente $P_{b}$ con la composición cosolvente

\begin{tabular}{|c|c|c|}
\hline \multicolumn{2}{|c|}{ PG } & \multirow[t]{2}{*}{$P_{b}$} \\
\hline$\% \mathrm{~m} / \mathrm{m}$ & $f$ & \\
\hline 0,00 & 0,0000 & 0,81 \\
\hline 10,00 & 0,0969 & 0,86 \\
\hline 20,00 & 0,1944 & 0,91 \\
\hline 30,00 & 0,2926 & 0,99 \\
\hline 40,00 & 0,3916 & 1,07 \\
\hline 50,00 & 0,4912 & 1,17 \\
\hline 60,00 & 0,5915 & 1,28 \\
\hline 70,00 & 0,6925 & 1,39 \\
\hline 80,00 & 0,7943 & 1,49 \\
\hline 90,00 & 0,8968 & 1,60 \\
\hline 100,00 & 1,0000 & 1,71 \\
\hline
\end{tabular}

De acuerdo con estos valores, se tiene también un cambio en las hidrofobicidades aparentes de los fármacos ya que por debajo del $30 \% \mathrm{~m} / \mathrm{m}$ de PG (aprox. $f 0,6$ ) el NAP sería más lipofílico que el IBP $\left(P_{b}<1\right)$, mientras que por encima de este valor se tendría el comportamiento contrario $\left(P_{b}>1\right)$, esto es, el IBP sería más lipofílico que el NAP. Lo observado mediante el análisis inmediatamente anterior es casi concordante con lo presentado previamente y referido a la Figura 1, con la única diferencia de las correspondientes fracciones de PG en las mezclas cosolventes, 0,4 y 0,6 , respectivamente. Esto podría en principio suponer un efecto de tipo camaleónico en las hidrofobicidades de los dos compuestos, lo cual se ha descrito en la literatura con el estudio de la solubilidad de algunos fármacos en diferentes mezclas solventes, y basado en la obtención de dos máximos de solubilidad en composiciones cosolventes de diferente polaridad ${ }^{28}$.

Un análisis más profundo de este comportamiento aparentemente anómalo de variación de las lipofilicidades de estos dos fármacos implicaría realizar otras determinaciones experimentales, entre las cuales destacarían análisis termodinámicos de transferencia involucrando no solamente los cambios de energías libres sino también las respectivas contribuciones entálpicas y entrópicas a los procesos, como ha sido realizado en el estudio de otros compuestos ${ }^{8,29}$, así como diferentes análisis de tipo espectroscópico para evaluar el comportamiento del medio solvente.

De otro lado, en la Figura 3 se presentan las desviaciones presentadas en la solubilidad de los dos fármacos respecto a lo estimado mediante la Ecuación 1, encontrándose desviaciones negativas para los dos compuestos en todo el intervalo de composición cosolvente. Este comportamiento es parcialmente diferente al presentado por Rubino y Obeng ${ }^{11}$ en el estudio de series homólogas de p-hidroxi- y p-aminobenzatos de alquilo, en los cuales se encontraron desviaciones negativas a bajas proporciones de PG y desviaciones positivas a altas proporciones del cosolvente. Como se indicó previamente, una explicación posible para las desviaciones negativas presentadas a bajas proporciones de cosolvente, podría hallarse en el trabajo de Kimura et al. ${ }^{14}$, quienes obtuvieron comportamientos similares en los calores de disolución de 1-metil2-pirrolidinona en mezclas alcohol-agua, y según el cual, a bajas concentraciones de cosolvente, el agua retiene su habilidad para formar estructuras ordenadas.

Como es bien sabido, los cosolventes poseen grupos funcionales polares y no polares en su estructura molecular y por lo tanto, se espera que la hidratación hidrofóbica ocurra alrededor de las partes no polares del compuesto, mientras que los enlaces de hidrógeno se forman entre los grupos polares y el agua. Aunque el PG ha sido generalmente considerado como un compuesto polar, Matsumoto et $a l^{7}$ han presentado evidencia de la influencia del grupo metilo terminal de este cosolvente sobre la estructuración del agua, mediante el estudio de las entalpías molares de exceso en mezclas PG-W. Estas interacciones existentes entre el PG y el agua podrían reducir la interacción entre el agua y los fármacos estudiados, llevando a una solubilidad inferior a la esperada según la Ecuación 1.

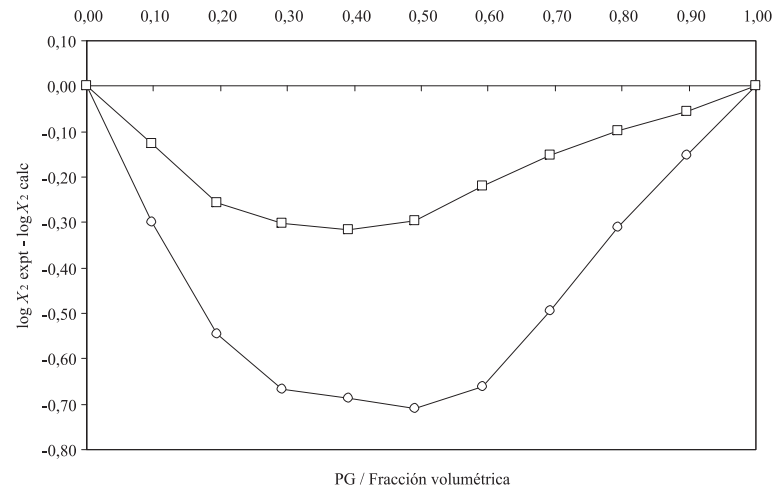

Figura 3. Desviaciones de la solubilidad experimental respecto a la linealidad para IBP (O) y NAP $(\square)$ a $25,0^{\circ} \mathrm{C}$

Según la Tabla 1 y la Figura 3, las máximas desviaciones se encuentran en fracciones 0,4 y 0,5 de PG para el NAP y el IBP, respectivamente, siendo todas las desviaciones, mucho más altas en magnitud para el IBP que para el NAP. Una posible explicación de esta última observación podría darse en términos de la presencia del grupo metoxilo en el NAP, el cual puede establecer enlaces de hidrógeno con el agua y con el PG favoreciendo las interacciones soluto-solvente mientras que el IBP debido a su grupo isopropilo induciría la autoasociación del agua para interactuar por hidratación hidrofóbica. Rubino y Obeng ${ }^{11}$ encontraron desviaciones a la Ecuación 1 cada vez mayores al incrementar la longitud de la cadena alquílica de p-hidroxi y p-aminobenzoatos, esto es, al aumentar la lipofilicidad de los compuestos. De acuerdo con lo anterior, podrían agregarse los resultados presentados en la Figura 3 a la reflexión de que el IBP es aparentemente más lipofílico que el NAP. Estos comportamientos podrían explicarse basados en el hecho de que la interacción entre el agua y un soluto lipofílico será más altamente dependiente de la disponibilidad de moléculas de agua para mantener al soluto en solución debido a la baja densidad de grupos formadores de enlaces de hidrógeno en el soluto, por lo que los compuestos más lipofílicos serán más sensibles a las interacciones cosolvente-agua. De otro lado, es posible que en el caso del IBP, adicionalmente se puedan estar presentando algunos fenómenos de naturaleza interfacial, puesto que recientemente se le han atribuido ciertas propiedades tensioactivas a este fármaco bajo determinadas condiciones experimentales ${ }^{30}$. 


\section{CONCLUSIONES}

De todo lo discutido anteriormente se tiene que el IBP y el NAP presentan desviaciones negativas al modelo logarítmico-lineal de Yalkowsky en el sistema binario PG-W, desviaciones que pueden en principio ser atribuidas a efectos de interacción solvente-solvente en las mezclas solventes, así como a las diferencias en la hidratación hidrofóbica de los solutos. Lo anterior hace impráctico el uso del modelo de aditividad de solubilidades para la estimación de esta propiedad fisicoquímica en el caso de estos dos fármacos en este sistema cosolvente, durante el diseño de formulaciones líquidas homogéneas. Así mismo, aparentemente se ha encontrado un efecto camaleónico de hidrofobicidades para los dos fármacos, siendo uno de los dos más hidrofóbico que el otro, dependiendo de la proporción de cosolvente en las diferentes mezclas. Finalmente, sería muy importante estudiar la solubilidad de otros AINEs derivados del ácido propiónico, tales como el ketoprofén, para poder ampliar el análisis de hidrofobicidad basado en esta propiedad fisicoquímica, como apoyo adicional a los estudios basados en coeficientes de reparto y otras propiedades termodinámicas de transferencia.

\section{AGRADECIMIENTOS}

Muy formalmente a la DIB-DINAIN de la Universidad Nacional de Colombia (UNC) por el apoyo financiero y al Departamento de Farmacia de la UNC por facilitar los equipos e instalaciones requeridos para el desarrollo de esta investigación.

\section{REFERENCIAS}

1. Roberts II, L. J.; Morrow, J. D. En Goodman \& Gilman's. The Pharmacological Basis of Therapeutics; $10^{\text {th }}$ ed.; Hardman, J. G.; Limbird, L. E.; Gilman, A. G., eds.; McGraw-Hill: New York, 2001, p. 703-5; Lund, W.; The Pharmaceutical Codex: Principles and Practice of Pharmaceutics, $12^{\text {th }}$ ed.; The Pharmaceutical Press: London, 1994

2. Rosenstein-Ster, E.; Diccionario de Especialidades Farmacéuticas, $32^{\mathrm{a}}$ ed.; Thompson P.L.M., S.A.: Bogotá, 2004.

3. Jiménez, F.; Martínez, F.; Rev. Col. Cienc. Quím. Farm. 1995, 24, 19.

4. Yalkowsky, S. H.; Roseman, T. J. En Techniques of Solubilization of Drugs; Yalkowsky, S. H., ed.; Marcel Dekker, Inc.: New York, 1981, p. 91-134.

5. Rubino, J. T. En Encyclopedia of Pharmaceutical Technology; Swarbrick, J.; Boylan, J. C., eds.; Marcel Dekker, Inc.: New York, 1988, vol. 3, p. 375-98.

6. Geyer, H.; Ulbig; P.; Görnert, M.; J. Chem. Thermodyn. 2000, 32, 1585; Geyer, H.; Ulbig, P.; Görnert, M.; Susanto, A.; J. Chem. Thermodyn. 2001, 33, 987; Jiménez, J.; Martínez, F.; Rev. Col. Cienc. Quím. Farm. 2005, 34, 46.

7. Matsumoto, Y.; Touhara, H.; Nakanishi, K.; Watanabe, N.; J. Chem. Thermodyn. 1977, 9, 801.
8. Jiménez, J. A.; Martínez, F.; J. Braz. Chem. Soc. 2006, 17, 125.

9. Martínez, F.; Rev. Acad. Colomb. Cienc. 2005, 29, 429; Manrique, Y. J.; Pacheco, D. P.; Martínez, F.; Resúmenes del XVII Congreso Farmacéutico Argentino, Villa Carlos Paz, Argentina, 2006.

10. Jouyban-Gharamaleki, A.; Valaee, L.; Barzegar-Jalali, M.; Clark, B. J.; Acree Jr., W. E.; Int. J. Pharm. 1999, 177, 101; Nokhodchi, A.; Shokri, J.; Barzegar-Jalali, M.; Ghafourian, T.; Farmaco 2002, 57, 555.

11. Rubino, J. T.; Obeng, E. K.; J. Pharm. Sci. 1991, 80, 479.

12. Rubino, J. T.; Yalkowsky, S. H.; Pharm. Res. 1987, 4, 220.

13. Yalkowsky, S. H.; Solubility and Solubilization in Aqueous Media, American Chemical Society and Oxford University Press: New York, 1999, p. 20-80; Millard, J. W.; Alvarez-Nuñez, F. A.; Yalkowsky, S. H.; Int. J. Pharm. 2002, 245, 153.

14. Kimura, F.; Murakami, S.; Fujishiro, R.; J. Solution Chem. 1975, 4, 241.

15. US Pharmacopeia, $28^{\text {th }}$ rev.; United States Pharmacopeial Convention, Inc.: Rockville, 2004.

16. Martínez, F.; Gómez, A.; Ávila, C. M.; Acta Farm. Bonaerense 2002, 21, 107; Anton Paar, K. G.: Calculating Digital Density Meter Instruction Manual, Graz: Austria, 1980, p. 1-12.

17. Manrique, Y. J.; Tesis de Maestría, Universidad Nacional de Colombia, Colombia, 2006; Pacheco, D. P.; Tesis de Maestría, Universidad Nacional de Colombia, Colombia, 2006.

18. Budavari, S.; O’Neil, M. J.; Smith, A.; Heckelman, P. E.; Obenchain Jr., J. R.; Gallipeau, J. A. R.; D'Arecea, M. A.; The Merck Index: An Encyclopedia of Chemicals, Drugs, and Biologicals, $13^{\text {th }}$ ed., Merck \& Co., Inc.: Whitehouse Station, 2001.

19. Betageri, G. V.; Nayernama, A.; Habib, M. J.; Int. J. Pharm. Adv. 1996, 1, 310.

20. Ertel, K.; Heasley, R. A.; Koegel, C.; Chakrabart, A.; Carstensen, J. T.; J. Pharm. Sci. 1990, 79, 52.

21. Perlovich, G. L.; Kurkov, S. V.; Kinchin, A. N.; Bauer-Brandl, A.; Eur. J. Pharm. Biopharm. 2004, 57, 411.

22. Burgot, G.; Burgot, J. L.; Ann. Pharm. Fr. 1995, 53, 13.

23. Lozano, H. R.; Martínez, F.; Quim. Nova 2006, 29, 704.

24. Mora, C. P.; Tesis de Maestría, Universidad Nacional de Colombia, Colombia, 2006.

25. Fedors, R. F.; Polym. Eng. Sci. 1974, 14, 147; Reillo, A.; Escalera, J. B.; Selles, E.; Ciencia Pharmaceutica 1993, 3, 249.

26. Ahumada, A. A.; Tesis Doctoral, Universidad Nacional de Córdoba, Argentina, 1987.

27. Manzo, R. H.; J. Pharm. Pharmacol. 1982, 34, 486; Manzo, R. H.; Anal. Asoc. Quím. Argent. 1983, 71, 47; Manzo, R. H.; Ahumada, A. A.; Acta Farm. Bonaerense 1984, 3, 3.

28. Martin, A.; Wu, P. L.; Liron, Z.; Cohen, S.; J. Pharm. Sci. 1985, 74, 638; Escalera, J. B.; Bustamante, P.; Martin, A.; J. Pharm. Pharmacol. 1994, $46,172$.

29. Bustamante, P.; Romero, S.; Reillo, A.; Pharm. Sci. 1995, 1, 505; Romero, S.; Reillo, A.; Escalera, B.; Bustamante, P.; Chem. Pharm. Bull. 1996, 44, 1061; Bustamante, P.; Romero, S.; Peña, A.; Escalera, B.; Reillo, A.; J. Pharm. Sci. 1998, 87, 1590; Jiménez, J. A.; Martínez, F.; J. Solution Chem. 2006, 35, 335 .

30. Stephenson, B. C.; Rangel-Yagui, C. O; Junior, A. P.; Tavares, L. C.; Beers, K.; Blankschtein, D.; Langmuir 2006, 22, 1514. 